\begin{tabular}{|c|l|}
\hline Title & Spatial mode demultiplexing technique using angularly multiplexed volume holograms with a phase plate \\
\hline Author(s) & $\begin{array}{l}\text { Shimizu, Shimpei; OKamoto, A tsushi; Mizukawa, Fumiya; Ogawa, Kazuhisa; Tomita, A kihisa; Takahata, Taketoshi; } \\
\text { Shinada, Satoshi; Wada, Naoya }\end{array}$ \\
\hline Citation & $\begin{array}{l}\text { Japanese Journal of A pplied Physics (JJAP), 56/9), 09NA 05 } \\
\text { https://doi.org/L0.7567/JJAP.56.09NA 05 }\end{array}$ \\
\hline Issue Date & 2017-09 \\
\hline Doc URL & http://hdl.handle.net/2115/71407 \\
\hline Rights & ○2017 The Japan Society of A pplied Physics \\
\hline Type & article (author version) \\
\hline File Information & JJAP_shimizu_final.pdf \\
\hline
\end{tabular}

Instructions for use 


\title{
Spatial mode demultiplexing technique using angularly multiplexed volume holograms with a phase plate
}

\author{
Shimpei Shimizu ${ }^{1 *}$, Atsushi Okamoto ${ }^{1,4}$, Fumiya Mizukawa ${ }^{1}$, Kazuhisa Ogawa ${ }^{1}$, \\ Akihisa Tomita ${ }^{1}$, Taketoshi Takahata ${ }^{2,4}$, Satoshi Shinada ${ }^{3}$, and Naoya Wada ${ }^{3}$ \\ ${ }^{1}$ Graduate School of Information Science and Technology, Hokkaido University, Sapporo \\ 060-0814, Japan \\ ${ }^{2}$ OPTOQUEST Co., Ltd., Ageo, Saitama 362-0021, Japan \\ ${ }^{3}$ National Institute of Information and Communications Technology (NICT), Koganei, Tokyo \\ 184-8795, Japan \\ ${ }^{4}$ OPTOQUEST Advanced Optical Device Development Research Division, Hokkaido \\ University, Sapporo 001-0021, Japan \\ *E-mail: s-shimizu@optnet.ist.hokudai.ac.jp
}

In this paper, we propose a spatial mode demultiplexing technique that uses a volume holographic demultiplexer (VHDM) with a phase plate. The VHDM can separate the multiplexed spatial modes with a single device by using angularly multiplexed volume holograms. In the VHDM, modal cross-talks, which are called inter-page cross-talks in the holographic data storages, are generated, and hence the separation performance of the VHDM is degraded by the intensity overlap between spatial modes. Therefore, we propose a novel scheme wherein phase modulation with a phase plate is added to the conventional scheme. The proposed scheme can achieve high separation performance by modulating the phase of spatial modes to reduce the intensity overlap. In this study, we demonstrated the basic operation of the proposed method for a specific linearly polarized mode group. The separation performance of the VHDM was observed to be markedly improved by the proposed method. 


\section{Introduction}

The transmission capacity of optical communication is facing its limitation because of the saturation of maximum incident power into single-mode fibers (SMFs) owing to the nonlinear Shannon limit and the fiber-fuse phenomenon. ${ }^{1-3)}$ On the other hand, there has been a significant increase in the growth of internet traffic recently. Therefore, the capacity crunch is rapidly approaching. ${ }^{4}$ To solve this problem, the mode division multiplexing (MDM) transmission in few-mode fibers (FMFs) has attracted considerable research attention. ${ }^{5-10)}$ The MDM treats each spatial mode in an FMF as an independent transmission channel. Moreover, it can be combined with conventional multiplexing methods such as wavelength division multiplexing. Thus, the MDM can greatly improve the transmission capacity in proportion to the number of spatial modes. In the MDM system, a mode demultiplexer is required in order to extract a specific signal from multiplexed signals, and various demultiplexing techniques have been proposed in this regard. ${ }^{11-19)}$ Above all, free-space optics schemes have received attention because of their extensibility in the number of multiplexed modes. However, the extension of the number of multiplexed modes leads to the cost escalation and the upsizing of the optical system in those schemes. Therefore, conventional free-space optics schemes are not suitable for the high integration of the MDM system and mass production.

For realizing a low-cost mode demultiplexer with a simple optical system, a volume holographic demultiplexer (VHDM) has been proposed. ${ }^{2021)}$ The VHDM utilizes angularly multiplexed volume holograms to separate multiplexed spatial modes. These holograms are often used to extract data in the holographic data storage (HDS). ${ }^{22,23)}$ In the HDS, the holograms are recorded as interference fringes between object beams containing data pages and reference beams, and then data pages are reconstructed by irradiating the reference beams to the holograms. In contrast, the VHDM uses object beams consisting of spatial modes to readout holograms, and then separates multiplexed spatial modes simultaneously. The cost and size of the VHDM are markedly lower than those of other free-space optics schemes because this scheme requires only one holographic medium, which is independent of the number of multiplexed modes. In the VHDM, each spatial mode is modulated with not only the hologram corresponding to it but also nonreconstruction holograms, which are holograms corresponding to other modes. Thus, modal cross-talks (MXTs), which are called 
interpage XTs in the HDS, are generated from nonreconstruction holograms. ${ }^{24)}$ Therefore, the mode separation performance of the VHDM is degraded because of the intensity overlap between spatial modes. Previous studies have indicated that the separation performance with a large overlap is lower than that with a small overlap, thereby supporting the aforementioned assertion. ${ }^{20,25)}$ However, in many cases of the MDM, near-order modes, wherein large overlaps exist, are expectedly used.

In this study, in order to improve the performance of the VHDM for near-order modes without significantly increasing the number of optical elements, we propose a VHDM combined with a phase plate. The proposed demultiplexer can improve the mode separation performance by converting the intensity distribution of each spatial mode using a phase plate. The profile of a phase plate depends on the combination of spatial modes for reducing the intensity overlap between them. Thus, each spatial mode can avoid modulating with nonreconstruction holograms. We demonstrated the basic operation of the proposed method for a specific linearly polarized (LP) mode group consisting of $\mathrm{LP}_{0,1}, \mathrm{LP}_{1,1}$, and $\mathrm{LP}_{2,1}{ }^{26)}$ The experimental results showed that the separation performance of the VHDM is considerably enhanced by the proposed method.

\section{Volume holographic demultiplexer with a phase plate}

Figure 1 shows the schematic diagrams of the proposed demultiplexer. The VHDM utilizes volume holograms consisting of interference fringes between spatial modes (separation targets) and reference beams (Gaussian beams) for mode demultiplexing. The incident angles of the reference beams are tilted in each recording, and then angularly multiplexed volume holograms are formed at the same region of the recording medium. By irradiating the holograms with the MDM signal, spatial mode components are diffracted as Gaussian beams to different angles and are spatially separated at each port in the SMF array. However, the MXTs are generated from the region where spatial modes are modulated with nonreconstruction holograms, that is, the overlap area between spatial modes and nonreconstruction holograms. Therefore, the amount of the overlap of intensity distributions between spatial modes affects the mode separation performance of the VHDM. In the proposed demultiplexer, the intensity conversion of spatial modes is conducted as a preprocessing in recording and demultiplexing procedures by phase modulation using a 
phase plate and the optical Fourier transform (OFT) via a lens. The phase plate needs to be designed so that the amount of intensity overlap between converted spatial modes could be low value. By performing this process, each spatial mode is mostly not modulated with nonreconstruction holograms, and thus, the proposed demultiplexer can achieve high separation performance.

In the following section, the diffraction property of the proposed demultiplexer is described based on Refs. 20 and 27. During the recording procedure [Fig. 1(a)], each spatial mode is sequentially generated, for example, by the spatial light modulator (SLM), and acts as the writing beam $W_{m}(m=1,2, \ldots, M)$, which is vertically incident on the recording medium. In the $m$-th recording, the writing beam $W_{m}$ and the reference beam $R_{m}$ are expressed as

$$
\begin{aligned}
& W_{m}=A_{m}(x, y, z) \exp \left[j k z+j \varphi_{m}(x, y, z)\right], \\
& R_{m}=A_{\mathrm{r}}(x, y, z) \exp \left[j k\left(x \sin \theta_{m}+z \cos \theta_{m}\right)\right],
\end{aligned}
$$

where $A_{m}(x, y, z)$ and $A_{\mathrm{r}}(x, y, z)$ are the amplitudes, $\theta_{m}$ is the incident angle, $\varphi_{m}(x, y, z)$ is the phase distribution of the spatial mode, and $k$ is the wave number in the recording medium. After applying the OFT via the first lens, the writing beam is modulated by the phase plate, which is placed on the Fourier plane, and modulated $W_{m}$ is expressed as

$$
W_{m}=F T\left[A_{m}(x, y, z) \exp \left[j k z+j \varphi_{m}(x, y, z)\right]\right] \exp \left[j \varphi_{p}(x, y, z)\right],
$$

where $\varphi_{\mathrm{p}}(x, y, z)$ is the phase profile of the phase plate. Then, by the OFT via the second lens, the intensity distribution of $W_{m}$ is converted according to the modulated phase. The converted writing beam $W_{m}{ }^{\prime}$ is expressed as

$$
\begin{aligned}
W_{m}{ }^{\prime} & =A_{m}(x, y, z) \exp \left[j k z+j \varphi_{m}(x, y, z)\right] * F T\left[\exp \left[j \varphi_{p}(x, y, z)\right]\right] \\
& =A_{m}{ }^{\prime}(x, y, z) \exp \left[j k z+j \varphi_{m}{ }^{\prime}(x, y, z)\right],
\end{aligned}
$$

where * denotes the convolution, and $A_{m}{ }^{\prime}(x, y, z)$ and $\varphi_{m}{ }^{\prime}(x, y, z)$ are amplitude and phase distribution after the conversion, respectively. In the VHDM, writing beams are used to readout holograms, and therefore, the complex amplitude transmittance $T$ for the demultiplexing of spatial modes is expressed as

$$
T=\sum_{m=1}^{M} A_{\mathrm{r}}(x, y, z) \overline{A_{m}}(x, y, z) \exp \left[-j \varphi_{m}{ }^{\prime}(x, y, z)+j k x \sin \theta_{m}+j k z\left(\cos \theta_{m}-1\right)\right],
$$

where $\bar{A}$ denotes the complex conjugate of $A$.

During the demultiplexing procedure [Fig. 1(b)], the MDM signal, wherein spatial 
modes corresponding to writing beams are multiplexed, is the output from an FMF and is collimated. Then, the phase plate and lenses convert each spatial mode component of the MDM signal in the same way as the recording procedure. The converted MDM signal $S$ is then expressed as

$$
S=\sum_{n=1}^{M} a_{n}(t) A_{n}{ }^{\prime}(x, y, z) \exp \left[j k z+j \varphi_{n}{ }^{\prime}(x, y, z)\right],
$$

where $a_{n}(t)$ are the time series signals. By irradiating the angularly multiplexed volume holograms with this signal, spatial mode components are diffracted as

$$
\begin{aligned}
D & =\eta \int_{0}^{L} T S d z \\
& =\eta \sum_{m=1}^{M} \sum_{n=1}^{M} \int_{0}^{L} a_{n}(t) \overline{A_{m}{ }^{\prime}} A_{n}{ }^{\prime} A_{\mathrm{r}} \exp \left[j\left(\varphi_{n}^{\prime}-\varphi_{m}{ }^{\prime}\right)+j k\left(x \sin \theta_{m}+z \cos \theta_{m}\right)\right] d z,
\end{aligned}
$$

where $L$ is the thickness of the recording medium and $\eta$ is the diffraction efficiency. When $n=m$, each spatial mode component is converted to a beam whose spatial phase is zero and is strongly diffracted in the direction of $\theta_{m}$. When $n \neq m$, that is, when mode fields are mismatched, each spatial mode component is weakly diffracted as an MXT component whose spatial phase is not zero. These MXT components are suppressed by reducing the intensity overlap using the phase plate. Consequently, the diffracted beam $D$ consisting of strong beams is observed as

$$
D=\eta \sum_{m=1}^{N} a_{m}(t) \int_{0}^{L}\left|A_{m}{ }^{\prime}\right|^{2} A_{\mathrm{r}} \exp \left[j k\left(x \sin \theta_{m}+z \cos \theta_{m}\right)\right] d z .
$$

As a result, each spatial mode component in the MDM signal is diffracted to different angles corresponding to the incident angle of the reference beam that was actually used for the recording of its mode. Moreover, the intensity of each diffracted mode is also converted to a Gaussian beam via the lens because of its spatial phase, and then the fundamental mode components are excited to the SMF arrays. Thus, the proposed demultiplexer can perform a highly accurate demultiplexing function by utilizing both the phase matching characteristics of a volume hologram and the conversion of the intensity distribution of spatial modes using the phase plate.

\section{Experiment}


To confirm the basic operation of the proposed demultiplexer, we performed an experiment on demultiplexing for a specific LP mode group comprising $\mathrm{LP}_{0,1}, \mathrm{LP}_{1,1}$, and $\mathrm{LP}_{2,1}$. In this experiment, the profile of the phase plate was set to the phase conjugation of $\mathrm{LP}_{2,1}$ for reducing the intensity overlap between these three spatial modes. Figure 2 shows the experimental setup. A photopolymer with a thickness of $400 \mu \mathrm{m}$ was used as the recording medium. ${ }^{28)}$ A diode-pumped solid-state (DPSS) laser at a wavelength of $532 \mathrm{~nm}$, which is within the sensitivity range of the photopolymer, was used as the light source. The laser beam was cleaned up and expanded using an objective lens, a pinhole, and an achromatic lens after passing through an isolator. Then, the beam was divided into a writing arm and a reference arm by using a polarizing beam splitter (PBS) and a half-wave plate (HWP), and the power densities of the two arms were adjusted to about $0.2 \mathrm{~mW} / \mathrm{cm}^{2}$ on the photopolymer.

In the writing arm, each spatial mode was sequentially generated by an intensity-type SLM (ISLM; HOLOEYE LC-R 1080) displaying computer-generated holograms (CGHs) on it. ${ }^{29)}$ The CGH is simply calculated using an interference pattern between a spatial mode field and a plane wave, which enables us to generate an accuracy mode field. Subsequently, a pinhole was used to extract the first-order diffracted beam from the CGH because the CGH also generates unnecessary diffracted beams. After generating the spatial mode, the intensity was converted by using a phase-type SLM (PSLM; SANTEC SLM-100-01-0002-01) as the phase plate and the lens. The converted beam was modulated to a vertically polarized light by a HWP in order to interfere with the reference beams and was made to enter into the photopolymer. In the reference arm, the beam diameter was adjusted to $5 \mathrm{~mm}$, which sufficiently covers the fields of the spatial modes. The incident angle of the reference beam was tilted by changing the angle of the mirror before the $4-f$ system, and these angles $\theta_{1}, \theta_{2}$, and $\theta_{3}$ were set to $39^{\circ}, 40^{\circ}$, and $41^{\circ}$, respectively. The angle distance was $1.0^{\circ}$, which is sufficient to satisfy the angular selectivity of $0.09^{\circ}$ that is estimated on the basis of a coupled wave theory. ${ }^{30)}$

In this experiment, exposure times were set to $30 \mathrm{~s}$ for all the holograms. After recording all the holograms, signal beams were sequentially irradiated onto the photopolymer along the same path as that of the writing beams during the recording procedure, and the diffracted beams were detected by a charge-coupled device (CCD). 
Furthermore, to compare the proposed VHDM with the conventional one in terms of the separation performance, we also performed the recording procedure and the demultiplexing procedure in the conventional case, which means that nothing was displayed on the PSLM. In addition, converted spatial modes were captured by the CCD replaced with the photopolymer in order to confirm the reduction of the intensity overlap between the three spatial modes.

\section{Results and discussion}

Figure 3 shows the profile of the phase plate and the intensity distributions of the spatial modes captured by the CCD with and without the phase modulation using the phase plate. Moreover, for the quantitative estimation of the amount of the intensity overlap between two modes, we define the overlap ratio (OR) as the proportion of the overlap area calculated as

$$
\mathrm{OR}=\frac{2 \sum_{x} \sum_{y} I_{\mathrm{o}}(x, y)}{\sum_{x} \sum_{y}\left\{I_{1}(x, y)+I_{2}(x, y)\right\}},
$$

where $I_{1}(x, y)$ and $I_{2}(x, y)$ are the intensity of two modes detected by the CCD in each pixel, and $I_{\mathrm{o}}(x, y)$ is the overlapping intensity calculated as

$$
I_{0}(x, y)=\min \left\{I_{1}(x, y), I_{2}(x, y)\right\} .
$$

Figure 4 shows the calculation results of the OR. These results indicated that the amount of OR was suppressed in any combinations of the spatial modes. Thus, it can be stated that the intensity overlap of spatial modes can be reduced by the proposed method.

Figure 5 shows the diffracted beams reconstructed by each incident spatial mode. By irradiating the multiplexed holograms with an LP mode, the mode is diffracted in three directions $\theta_{i}(i=1,2,3)$ corresponding to the reference angles as a signal component and two XT components. Each diffracted beam reconstructed by the input of the $m$-th $(m=1,2$, 3 ) mode is denoted as $D_{m, i}$. Each intensity image of diffracted beam captured by the CCD is trimmed into a size of $50 \times 50$ pixels. The separation ratio $(\mathrm{SR})$ indicates the mode separation performance of the VHDM. The SR of the $m$-th mode recorded with the reference angle of $\theta_{i}$ is calculated as

$$
\mathrm{SR}_{m, i}=10 \log _{10}\left(\frac{P_{m, i}}{\sum_{n \neq m} P_{n, i}}\right),
$$


where $P_{m, i}$ is the optical power of $D_{m, i}$, which can be calculated with the sum of the value in each pixel. We can see that each mode component is strongly diffracted as a Gaussian beam at the corresponding angle in each case. Furthermore, the proposed method can improve the SR up to $2.93 \mathrm{~dB}$ compared with that of the conventional VHDM. In addition, Fig. 6 shows the MXT characteristics, which are the optical power of MXTs normalized by that of the signal component in each diffraction angle. These results showed that the MXTs have significantly improved between $\mathrm{LP}_{0,1}$ and $\mathrm{LP}_{2,1}$, which is the most effective combination for the reduction of OR. Moreover, a similar correlation between the power of MXTs and the OR has also been observed between the other modes. These results confirmed that the reduction of the intensity overlap between the recorded spatial modes is effective for improving the mode separation performance of the VHDM.

This experiment was performed using a phase plate whose profile is obtained from the phase distributions of LP modes. Therefore, the proposed method is expected to have higher performance if the phase plate can be optimized so that the overlap between modes is further reduced.

\section{Conclusions}

In this study, we proposed a mode demultiplexing technique based on the VHDM with a phase plate. The proposed method can improve the separation performance of VHDM by modulating the phase of spatial modes using a phase plate in order to reduce the intensity overlap between them. To confirm the basic operation of this method, the mode demultiplexing experiment was conducted using three spatial modes. The experimental results revealed that the three modes were separated along different angles, and the separation performance of the VHDM was improved when the intensity overlap of spatial modes was reduced. In the future, to realize higher performance and to apply the proposed method to arbitrary mode groups, we intend to investigate an optimization technique for the profile of the phase plate. 


\section{References}

1) R. J. Essiambre and R. W. Tkach, Proc. IEEE 100, 1035 (2012).

2) M. Secondini and E. Forestieri, Opt. Fiber Commun. Conf. (OFC) 2016, Th3D.1.

3) R. Kashyap and K. J. Blow, Electron. Lett. 24, 47 (1988).

4) D. J. Richardson, Science 330, 327 (2010).

5) H. Kubota and T. Morioka, Opt. Fiber Technol. 17, 490 (2011).

6) H. R. Stuart, Science 289, 281 (2000).

7) S. Randel, R. Ryf, A. Sierra, P. J. Winzer, A. H. Gnauck, C. A. Bolle, R. J. Essiambre, D. W. Peckham, A. McCurdy, and R. Lingle, Opt. Express 19, 16697 (2011).

8) V. A. J. M. Sleiffer, P. Leoni, Y. Jung, J. Surof, M. Kuschnerov, V. Veljanovski, S. U. Alam, D. J. Richardson, L. G. Nielsen, Y. Sun, B. Corbett, R. Winfield, S. Calabrò, and H. de Waardt, Opt. Express 22, 749 (2014).

9) J. Sakaguchi, W. Klaus, J.-M. D. Mendinueta, B. J. Puttnam, R. S. Luis, Y. Awaji, N. Wada, T. Hayashi, T. Nakanishi, T. Watanabe, Y. Kokubun, T. Takahata, and T. Kobayashi, Opt. Fiber Commun. Conf. (OFC) 2015, Th5C.2.

10) K. Igarashi, D. Soma, Y. Wakayama, K. Takeshima, Y. Kawaguchi, N. Toshikane, T. Tsuritani, I. Morita, and M. Suzuki, Opt. Express 24, 10213 (2016).

11) N. Hanzawa, K. Saitoh, T. Sakamoto, T. Matsui, K. Tsujikawa, M. Koshiba, and F. Yamamoto, Opt. Express 21, 25752 (2013).

12) S. H. Chang, H.S. Chung, N. K. Fontaine, R. Ryf, K. J. Park, K. Kim, J. C. Lee, J. H. Lee, B. Y. Kim, and Y. K. Kim, Opt. Express 22, 14229 (2014).

13) D. Sperti, M. Salsi, C. Koebele, P. Tran, H. Mardoyan, S. Bigo, A. Boutin, P. Sillard, and G. Charlet, Eur. Conf. Exhib. Opt. Commun. (ECOC) 2011, Th.12.B.2.

14) C. Koebele, M. Salsi, D. Sperti, P. Tran, P. Brindel, H. Mardoyan, S. Bigo, A. Boutin, F. Verluise, P. Sillard, M. Astruc, L. Provost, F. Cerou, and G. Charlet, Opt. Express 19, 16593 (2011).

15) A. Okamoto, K. Aoki, Y. Wakayama, D. Soma, and T. Oda, Opt. Fiber Commun. Conf. (OFC) 2012, JW2A.38.

16) V. A. J. M. Sleiffer, Y. Jung, V. Veljanovski, R. G. H. van Uden, M. Kuschnerov, H. Chen, B. Inan, L. G. Nielsen, Y. Sun, D. J. Richardson, S. U. Alam, F. Poletti, J. K. Sahu, A. Dhar, A. M. J. Koonen, B. Corbett, R. Winfield, A. D. Ellis, and H. de Waardt, Opt. Express 20, 
B428 (2012).

17) K. Igarashi, D. Souma, T. Tsuritani, and I. Morita, Opt. Express 22, 20881 (2014).

18) G. Labroille, B. Denolle, P. Jian, P. Genevaux, N. Treps, and JF. Morizur, Opt. Express 22, 15599 (2014).

19) K. Igarashi, D. Souma, K. Takeshima, and T. Tsuritani, Opt. Express 23, 183 (2015).

20) Y. Wakayama, A. Okamoto, K. Kawabata, A. Tomita, and K. Sato, Opt. Express 21, 12920 (2013).

21) K. Kawabata, A. Okamoto, S. Honma, Y. Wakayama, K. Sato, and A. Tomita, Proc. SPIE 8647, 86470C (2013).

22) D. Psaltis and G. W. Burr, Computer 31, 52 (1998).

23) F. H. Mok, Opt. Lett. 18, 915 (1993).

24) A. Shibukawa, A. Okamoto, Y. Wakayama, A. Tomita, H. Funakoshi, and K. Sato, Jpn. J. Appl. Phys. 53, 09LD03 (2013).

25) T. Oda, A. Okamoto, D. Soma, A. Tomita, and Y. Wakayama, Proc. SPIE 7958, 79580J (2011).

26) D. Gloge, Appl. Opt. 10, 2252 (1971).

27) E. G. Ramberg, RCA Rev. 27, 467 (1966).

28) J. Ikeda, R. Arai, N. Morishita, C. Katahira, Y. Takatani, S. Yumoto, K. Yokouchi, Y. Hayashi, Y. Tanaka, K. Watanabe, P. B. Lim, and M. Inoue, Int. Workshop Holographic Memories and Display (IWHM\&D) 2009, 3A-4.

29) F. Dubois, P. Emplit, and O. Hugon, Opt. Lett. 19, 433 (1994).

30) H. Kogelnik, Bell Syst. Tech. J. 48, 2909 (1969). 


\section{Figure Captions}

Fig. 1. (Color online) Schematic diagram of volume holographic demultiplexer with a phase plate. (a) Recording procedure. Writing beams $W_{i}$ converted by a phase plate are incident on a recording medium sequentially with reference beams $R_{i}$ to record holograms. (b) Demultiplexing procedure. By irradiating the MDM signal $S$, which is converted by a similar way to the recording procedure, to holograms, each mode component is diffracted along different angles, which are depicted as the diffracted beams $D$.

Fig. 2. (Color online) Experimental setup for demultiplexing of three modes. An ISLM (HOLOEYE LC-R 1080) generated spatial modes by displaying CGHs. A PSLM (SANTEC SLM-100-01-0002-01) was used as a phase plate to modulate the phase of spatial modes. The photopolymer with a thickness of $400 \mu \mathrm{m}$ was used as the recording medium.

Fig. 3. Profile of phase plate and intensity distributions of spatial modes with and without conversion by phase modulation using the phase plate.

Fig. 4. Overlap ratio of different combinations of two modes with and without intensity conversion by a phase plate.

Fig. 5. Images of diffracted beams for conventional and proposed VHDM when each spatial mode is irradiated to the recording medium. The SR indicates the separation performance of VHDM.

Fig. 6. MXT characteristics. The optical powers of MXTs normalized by the signal component for each diffraction angle are evaluated. (a) Results of conventional VHDM. (b) Results of proposed VHDM. 


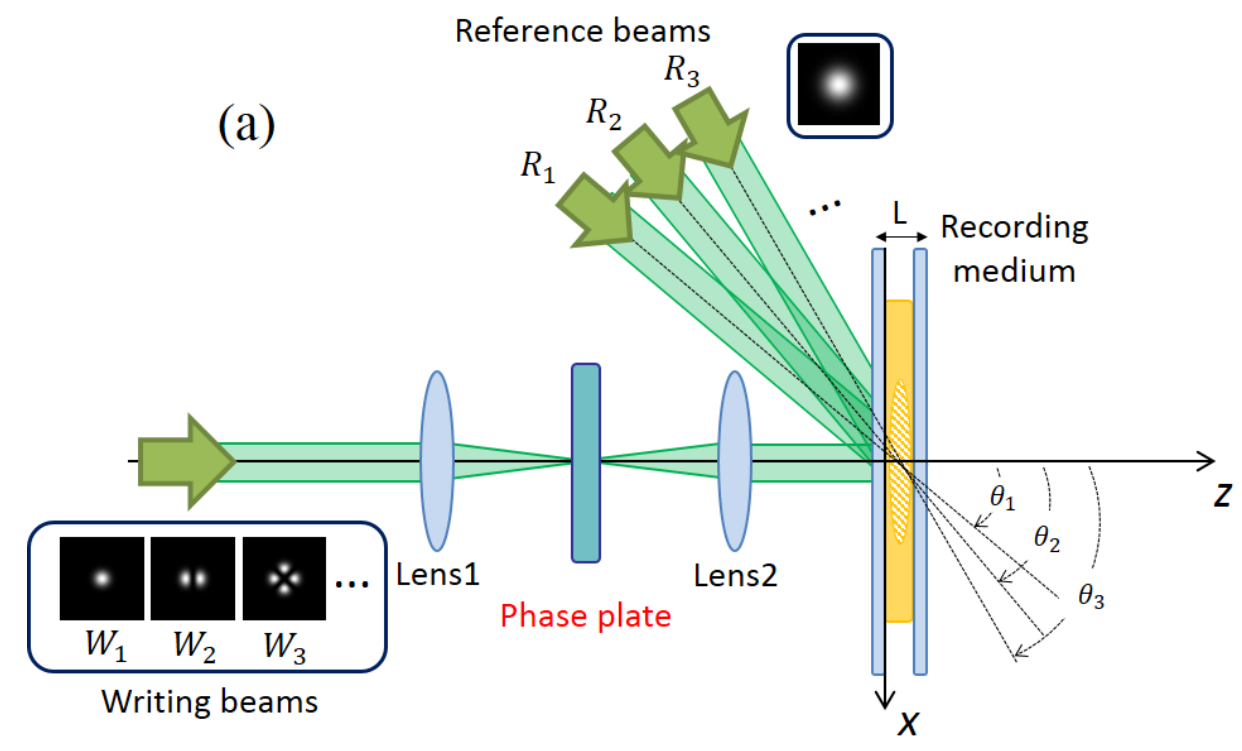

(b)

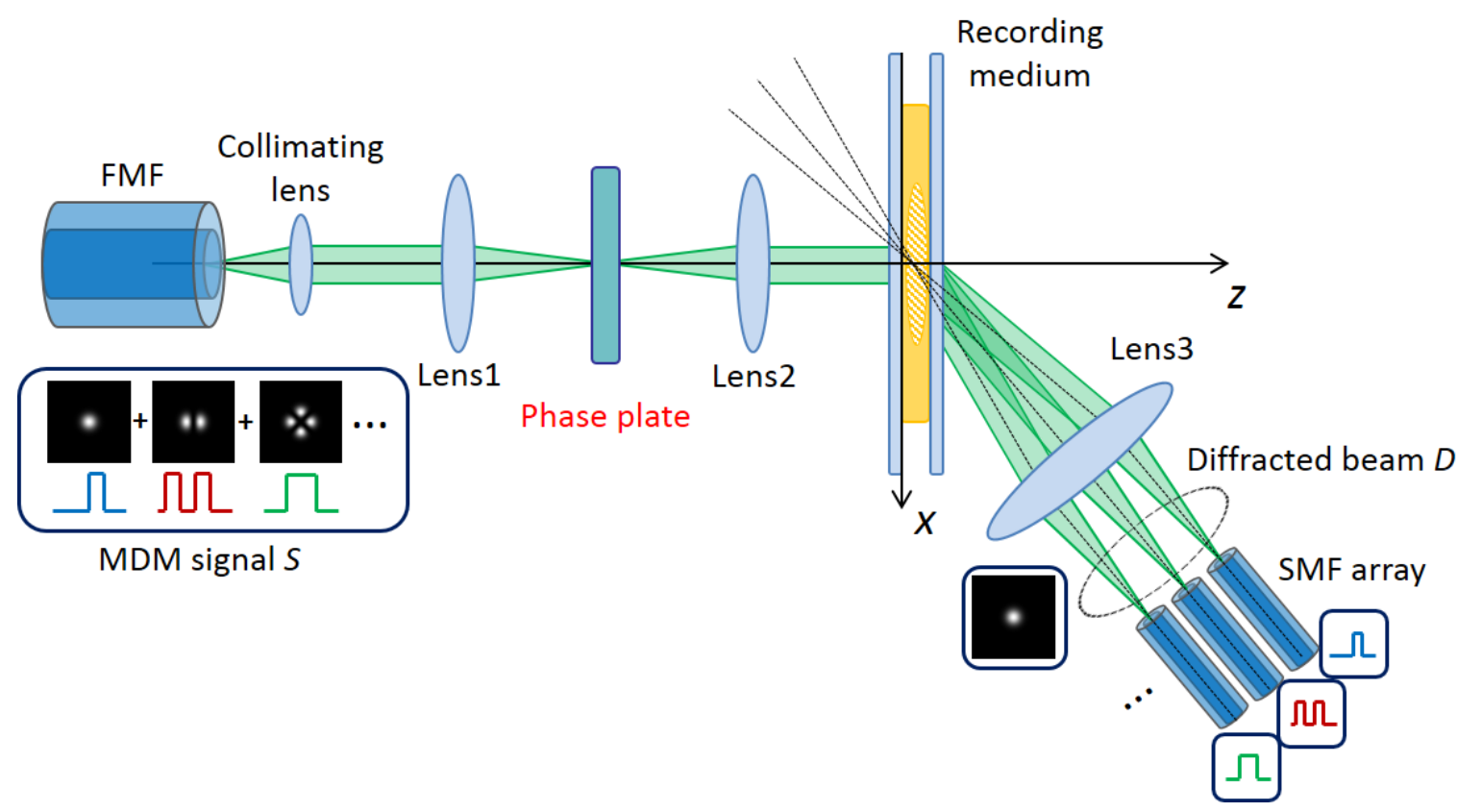

Fig. 1 (Color online) 


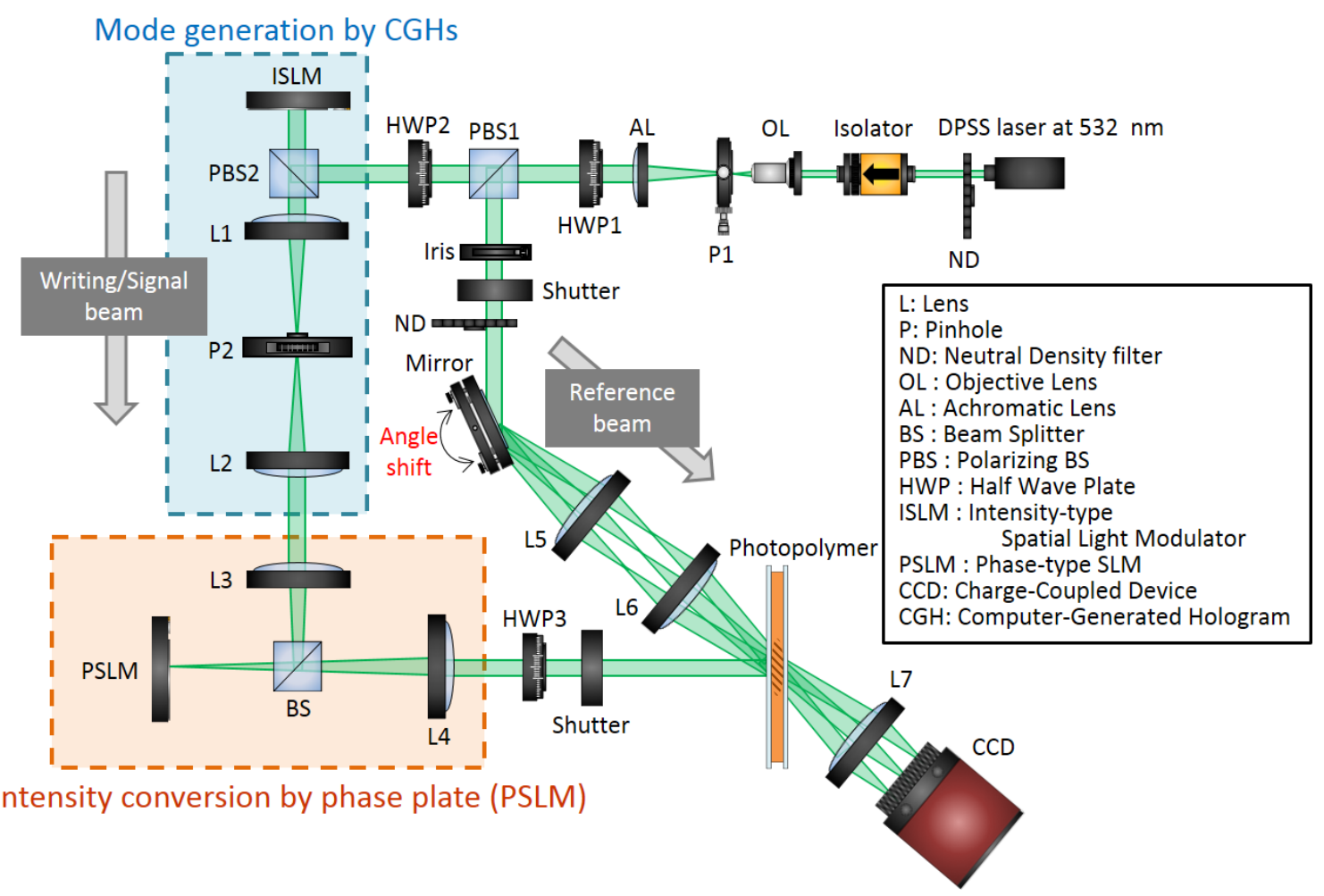

Fig. 2 (Color online) 


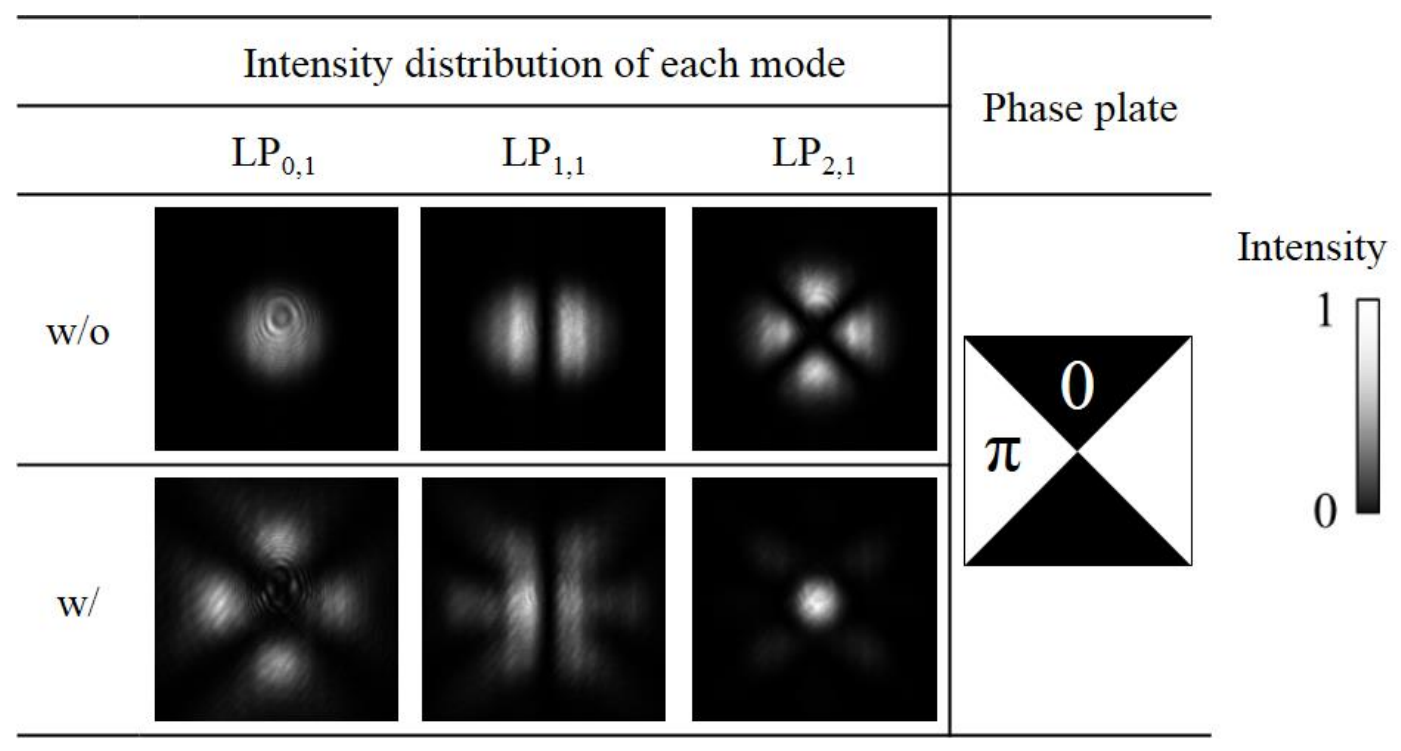

Fig. 3 


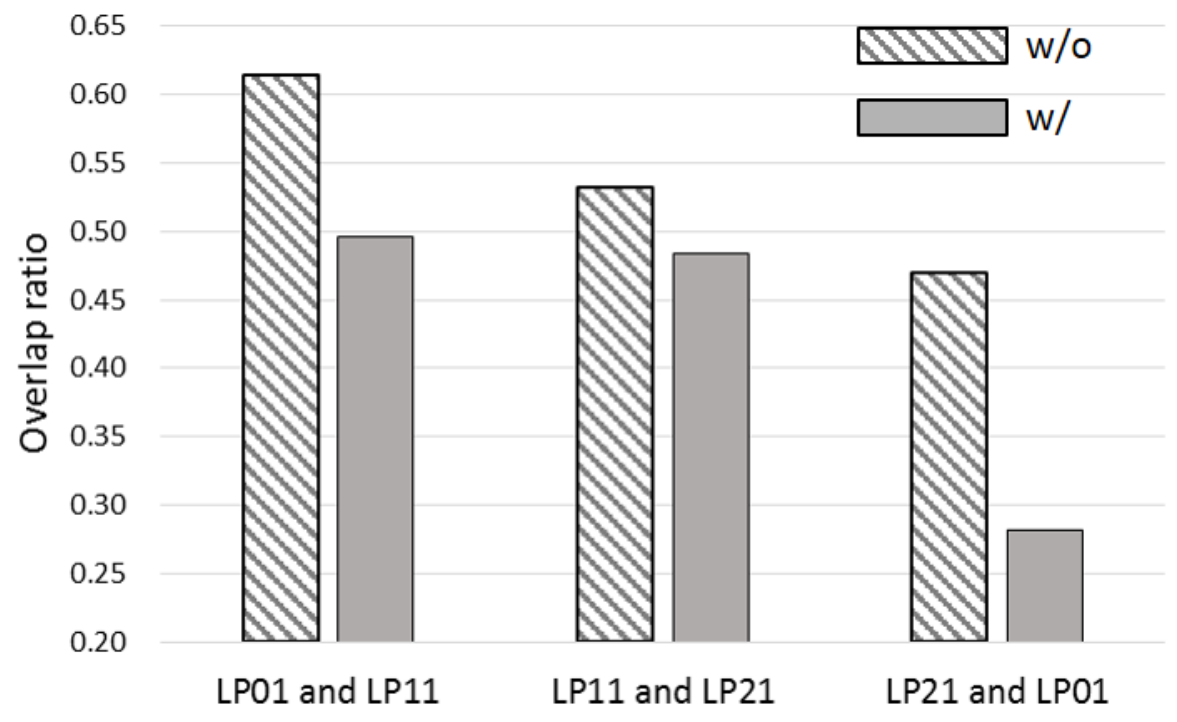

Fig. 4 


\begin{tabular}{|c|c|c|c|c|c|c|}
\hline & Cor & tional & $\mathrm{DM}$ & \multicolumn{3}{|c|}{ Proposed VHDM } \\
\hline & \multicolumn{6}{|c|}{ Diffraction angle } \\
\hline & $\theta_{1}$ & $\theta_{2}$ & $\theta_{3}$ & $\theta_{1}$ & $\theta_{2}$ & $\theta_{3}$ \\
\hline $\mathrm{LP}_{0,1}$ & $D_{1,1}$ & 1,2 & P,3 & $\overline{D_{1,1}}$ & 1,2 & \\
\hline $\mathrm{LP}_{1,1}$ & $D_{2,1}$ & 2,2 & & $D_{2,1}$ & $*$ & 2,3 \\
\hline $\mathrm{LP}_{2,1}$ & $D_{3,1}$ & 3 & \% & $\overline{\overline{D_{3,1}}}$ & 3,2 & 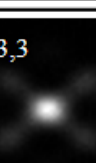 \\
\hline $\mathrm{SR}[\mathrm{dB}]$ & -1.31 & -1.47 & -1.02 & -0.14 & -0.52 & 1.91 \\
\hline
\end{tabular}

Fig. 5 
(a)

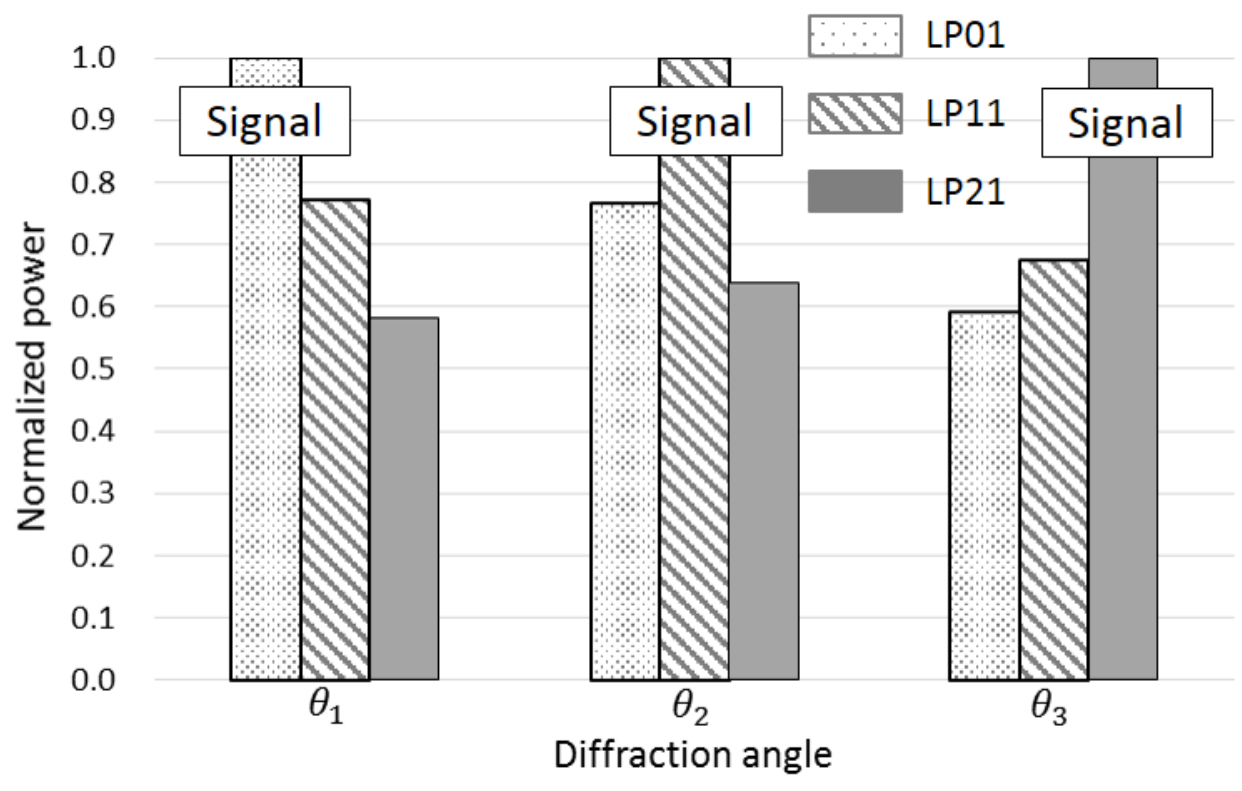

(b)

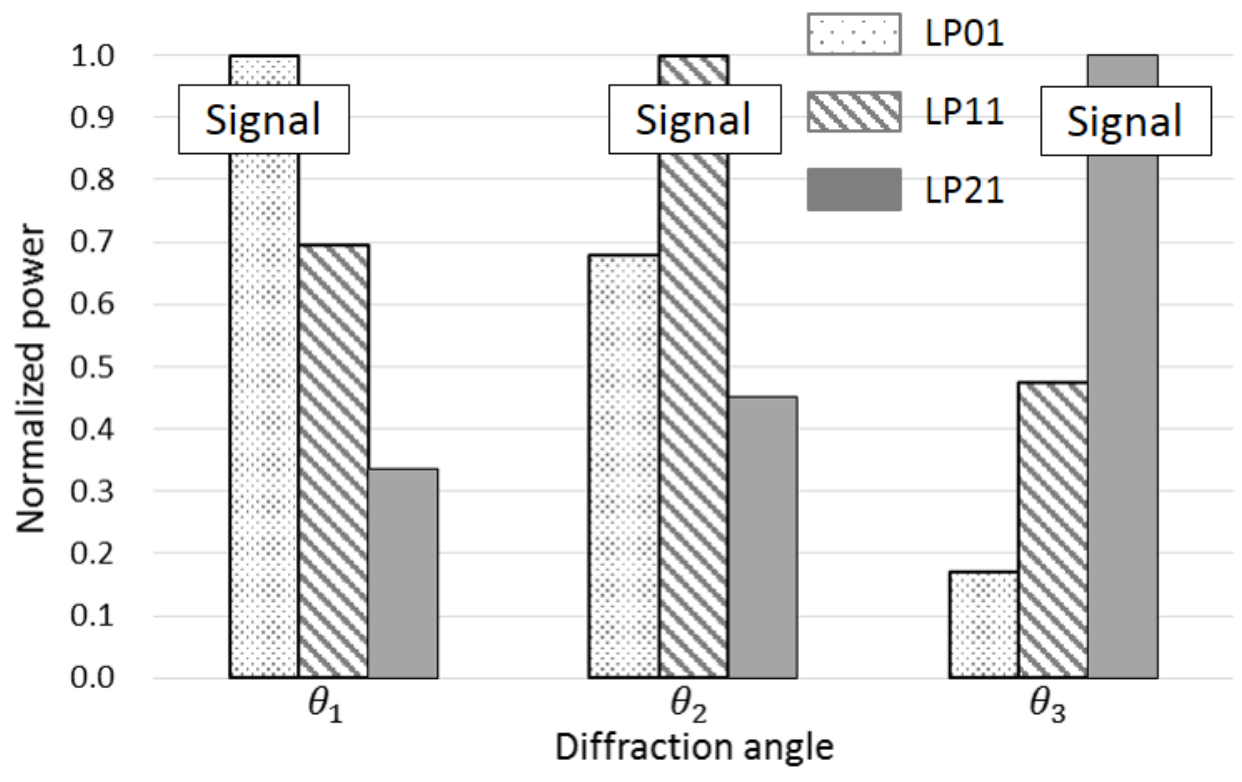

Fig. 6 